\title{
SCIENTIFIC REALISM AND THE CRITERIA FOR THEORY-CHOICE
}

\begin{abstract}
The central terms of certain theories which were valued highly in the past, such as the phlogiston theory, are now believed by realists not to refer. Laudan and others have claimed that, in the light of the existence of such theories, scientific realism is untenable. This paper argues in response that realism is consistent with - and indeed is able to explain - such theories' having been highly valued and yet not being close to the truth. It follows that the set of highly-valued past theories cited by Laudan, presumed to militate against realism, is in fact innocuous to the doctrine. The argument hinges largely on identifying the grounds on which theory-adoption is actually performed.
\end{abstract}

\section{A CHALlenge TO REALism}

Scientific realism associates the observational success of a theory to its truth. In its typical formulations, the doctrine maintains that a theory's having observational success to the highest possible degree is grounds for believing it to be true, and that a theory's having observational success to a somewhat lesser degree is grounds for believing it to be to a similar degree close to the truth. I take 'empirical adequacy', another expression which has occurred in the literature, to be synonymous with 'observational success'.

The requirement that a theory should possess a high degree of observational success is very onerous. A theory has observational success to the highest possible degree just if all its claims are true of all observable phenomena, including phenomena in the past and phenomena in other ways inaccessible to us; a theory has such-and-such a degree of observational success just if a corresponding proportion of its claims is true. This usage of 'observational success' is entrenched in the literature, among both antirealists and realists. (See respectively van Fraassen 1980 , p. 12, and Churchland 1985, pp. 38-39.)

The debate over realism cannot be understood unless it is appreciated that it is high degrees of observational success, and not some lesser or other properties of theories, which realists claim are grounds for believing a theory to be close to the truth. Clearly, the terms and outcome of the debate would be different if realists were positing an association between truth and some property of theories other than a high degree 
of observational success. For instance, if realists claimed that grounds for considering a theory to be close to the truth were provided by the theory's being consistent with some small set of previously-known data, realism would be very implausible, in the light of the ease with which false premises can be constructed to entail true conclusions of limited scope.

The degree of a theory's observational success is a property of it which depends only on the claims of the theory and the way the world is; in particular, it is independent of the degree of scientific attainment of those who embrace or consider the theory. A theory's degree of observational success does not vary with, for instance, the accumulation of evidence by a particular scientist or community. It is therefore strictly inappropriate to speak of a theory's 'having had' such-and-such a degree of observational success at a particular time, or of its 'having been' observationally very successful, if these phrases are meant to suggest that the theory might at a different time come to have some other degree of observational success.

It roughly follows from the realists' claims that the policy of choosing theories in virtue of the degrees of their observational success results in the adoption of theories which are close to the truth; though perhaps this result is obtained only once a certain stage has been reached in the history of a science, so that the science can be presumed to have reached 'maturity', or to have 'latched on' correctly to the structure of the world. Physical science since the late Renaissance is cited as the paradigmatic 'mature science'. (Formulations of these realist tenets may be found in Putnam 1978, pp. 20-21; Hesse 1980, pp. 154-155; NewtonSmith 1981, pp. 39-43; Boyd 1984, pp. 41-42.)

There is a class of historical instances which is purported to militate against scientific realism, or at least the version of the doctrine which I have reproduced above. Each of the episodes takes place in what was arguably a mature science, and has the following form: a theory to which the scientific community had acquired a commitment is discredited and succeeded by one containing theoretical terms radically different from those of its predecessor. Examples of these episodes are the substitution of theories appealing to phlogiston, caloric, or ether by later chemical, thermodynamic and electromagnetic theories. (Further historical examples are discussed in Laudan 1981, p. 33.) Let such episodes be called 'radical transitions'; and call the theories which 
were abandoned and adopted in the course of a radical transition the transition's 'earlier' and 'later' theories respectively.

Upon these episodes is based the following argument against scientific realism, first advanced by Laudan (1981, pp. 32-36, and 1984a, pp. 120-124). Radical transitions have occurred in mature sciences. Today's realists typically believe the later theories of many radical transitions to be 'close to the truth'. But in every radical transition, the entities posited by the transition's earlier theory are very different from those posited by the later theory: it would be inadmissible to believe in the existence of the entities of both theories. This means that realists must believe the transitions' earlier theories to be far from the truth, in any reasonable construal of 'proximity to the truth'. But the earlier as well as the later theory in each transition had demonstrated observational success to a considerable degree; indeed, the earlier theory as well as the later was chosen for adoption by the community on the strength of its possession of a high degree of observational success. It ensues that there are some theories in the mature sciences showing a high degree of observational success which realists, contrary to their own doctrine, are compelled to consider far from the truth. (It ensues similarly that the policy of choosing theories in virtue of their observational success has in the past resulted in the adoption of theories which the realist is compelled to consider far from the truth.) Therefore the observational success of theories cannot be associated with proximity to the truth in the way which realism envisages: we should do better to abandon the realist construal of theories, and consider the unobservable entities to which theories appeal as no more than useful fictions.

This argument has been acknowledged as a substantial challenge to scientific realism by several realists, among whom are Brown (1985, pp. 54-55), Miller (1987, pp. 399-400), and Newton-Smith (1990, p. 188). So far, most realists who have attempted to answer it have followed a particular strategy: they have accepted that the earlier theory in many radical transitions has a high degree of observational success, but denied that the realist is compelled to consider it far from the truth. For instance, Hardin and Rosenberg (1982) suggest that theories can be said to be approximately true even if their central terms do not refer; McMullin (1984, pp. 17-18) portrays the belief which was abandoned in typical radical transitions as not a proper scientific theory, but merely an intuitive assumption about the reality underlying phenomena, and 
thus not a doctrine of the sort which the realist is committed to seeing as true or false; Cummiskey (1992, pp. 33-37) appeals to a metaphortheory of reference to explain why the theoretical terms of a theory can fail to refer without the theory's being far from the truth. The strategy underlying these answers carries its own cost for realism, watering down the notion of proximity to the truth.

In this paper, I offer an answer to Laudan's argument which follows a strategy different from the above. I accept that the realist is committed to the claim that a theory's having a high degree of observational success is grounds for considering it close to the truth; and I accept that the earlier theories in the radical transitions cited by Laudan cannot plausibly be considered close to the truth. But I deny that the earlier theory in each of the radical transitions cited by Laudan has a high degree of observational success, however highly it was valued by its own proponents. I draw attention first to the nature of the criteria actually used in theory-appraisal in the history of science.

\section{DISCOVERING THE PROPERTIES OF THE PROPERTIES OF THEORIES}

To ascertain what criteria have actually been employed by scientists in selecting the theories which were to be legitimated as 'successful' is obviously relevant to a discussion of radical transitions. In that discussion, after all, the realist is called to explain how certain theories, preserved in the historical record as 'successful', can be (as the realist would put it) distant from the truth.

Philosophers who debate the plausibility of realism - realists as well as antirealists - frequently assume without second thought that theories which were in history counted as successful were judged to be so on the criteria reconstructed and prescribed by today's philosophers of science. In particular, since most of the participants to the current debate concur in considering degree of observational success to be one of the most notable properties of a theory, they tend to assume that scientists have judged theories - and judged certain theories to be successful - by checking their degrees of observational success.

Laudan himself seems to assume this, in presenting his challenge to realism. He writes for instance that "what the history of science offers us is a plethora of theories which were both successful and [ ...] nonreferential" (1981, p. 33). He clearly means to suggest that these theo- 
ries have been shown to possess a high degree of observational success: it is only under this assumption that realism can be confronted, as he intends it to be, with the challenge of explaining how theories which are observationally very successful can be far from the truth.

Drawing inspiration from Laudan, many subsequent authors have attributed high degrees of observational success to theories which fail to refer, heightening the seeming hopelessness of the realists' position. For instance, Thagard (1988, p. 149) writes: "Laudan [ . . ] shows that it is problematic to explain the success of particular theories by the assumption that they refer to the real world, for there have been successful theories, such as the wave theory of light with its luminiferous ether, that failed to refer." Thagard presumably supposes that, in its heyday, the luminiferous-ether theory was shown to possess a high degree of observational success: this is the only sort of 'success' which would make this theory a problem for realism, in view of the fact that this is the only sort of success to which statements of the realist doctrine allude.

If doubt could be cast upon the belief that such non-referring theories adopted in history indeed possess high degrees of observational success, the antirealist challenge would lose its effectiveness. This is what I propose to do. I intend first to undermine the assumption that scientists have ever judged theories to be successful or unsuccessful by checking their degree of observational success. Once this is done, it will be seen that the fact that a theory is legitimated in the historical record as 'successful' does not establish that it possesses a high degree of observational success. I will thereafter seek to suggest that, on the best evidence available today, the degree of observational success of many past theories, such as the earlier theories of radical transitions, is low, not high.

There are many ways in which the assumption that scientists judge theories by checking their degrees of observational success could be brought into question. An easy way would be by compiling an account of theory-appraisal which minimized the incidence of logical and empirical concerns of scientists and emphasized the role of their extra-empirical interests. (For a review of historical evidence that extra-empirical concerns operate in science, see e.g. Buchdahl 1970, pp. 204-206.) Suppose for instance that it could be plausibly argued that scientists saw as their goal the achievement of certain aesthetic or ethical ends, and therefore evaluated theories not for their empirical virtues but for 
nothing other than their aesthetic properties or ethical implications. Then it would appear plausible to conclude that scientists made only minimal appeal in their theory-evaluations to theories' degrees of observational success.

However, the cost of this route to the scientific realist would be unacceptable: the realist is committed to the theses that science has cognitive goals and consequently that procedures of theory-evaluation in at least the mature sciences have, in some sense, a grip on phenomena. While I wish to cast doubt on the assumption that theory-appraisal is conducted by reference to degrees of observational success, my ultimate aim is to defend realism: I must therefore seek a way of casting doubt on the assumption which does less harm than this to realism. I now sketch a model of theory-evaluation which refers to two sets of properties: the possible properties of theories, and these properties' own properties.

Theories have many possible properties. Among them (though not necessarily among the most interesting) are the property of being mathematical, the property of according with the data of a certain set, and the property of being susceptible of concise formulation. These properties of theories themselves have properties: for instance, it may be a property of one possible property of theories that different theories can possess it to different degrees, or that its presence in a theory is difficult to ascertain, or that it reveals itself in a theory only once the theory has been applied in the design of experiments.

Norms of theory-evaluation attach value to some properties of theories. They are prescriptions of the form: "Ceteris paribus, value a theory more highly if it possesses property $P$." Whether value ought to be, or can usefully be, attached by the norms of theory-evaluation to a particular property $P$ depends on (among other things) the properties of $P$. Among the properties of $P$ which carry the most weight in determining whether value should be attached to it by the norms of theory-evaluation are two: its logical relation with the goals attributed to science, and the ease with which its presence might be ascertained in theories. The properties which can most usefully be given value by the norms of theory-evaluation are those which are functionally related to the goals attributed to science, and are easily detected in theories.

Let us apply this analysis to a particular possible property of theories, observational success. In science, one often hears, theories are to be valued in accordance with their possession of observational success. 
(Realists and antirealists would differ in their interpretation of this maxim, since the former see the observational success of a theory as a by-product of its truth while the latter see the aim of science as observational success tout court; even so, both schools would be able to endorse this principle.) I agree that the property of observational success is what makes some theories valuable, and some theories preferable to others. But I deny that it is possible to choose among theories by checking a theory's possession of the property of observational success. My reasons refer to some properties of the property of observational success.

The property of observational success is such that a theory would be revealed to demonstrate the highest degree possible of observational success just if it were seen to accord with all data accumulated from all sources over unlimited time-spans; it would be revealed to demonstrate such-and-such a degree of observational success presumably only by being seen to accord with a corresponding proportion of such data. Checking, or obtaining a direct reading or measure of the degree of a theory's observational success (henceforth to be called the theory's 'OS measure'), would therefore presumably involve ascertaining the proportion of such data with which the theory accords.

It follows from this that it is not the case that the policy of evaluating and endorsing theories on the basis of OS measures has been pursued, or even could have been pursued, in the history of science. Even if the notion of counting and comparing the numbers of a theory's confirmed and disconfirmed predictions could be made precise, an OS measure could not be obtained in a finite time for generalizations of wide scope other than tautologies or contradictions. Scientists therefore cannot let theory-choices hang upon OS measures. (Another reason for doubting that our theory-appraisals are determined by OS measures is that, if they were, and if the OS measures were compiled correctly from the first, no new evidence could ever compel revisions of our past assessments of theories; and this suggestion seems contrary to our understanding of empirical science.)

Rather, scientists conduct theory-evaluation by the use of criteria able to indicate in a finite and, whenever possible, short time which theory among several available should be embraced or pursued. These criteria of course cite some of the possible properties of theories: but not, at least directly, their property of observational success.

The policy available to scientists which most closely approximates to 
the policy of evaluating theories on the basis of OS measures is that of judging theories for properties which are diagnostic of high OS measures. A property of a theory is diagnostic of high OS measures just if its possession by a theory indicates that the theory's OS measure is likely to be high. To the extent to which any property is diagnostic of high OS measures, a theory which exhibits it can legitimately be valued on the grounds that it is likely to possess a high OS measure.

Some properties of theories are more strongly diagnostic of high OS measures than others are: that is, there will be a stronger correlation between high OS measures and the possession of some properties than between high OS measures and the possession of others. Scientists who value the observational success of theories should, of course, strive to identify properties of theories as strongly diagnostic of high OS measures as possible, for use in their criteria of theory-evaluation.

The strength with which criteria formulated by scientists are diagnostic of high OS measures depends on the scientists' awareness and understanding of the properties of possible properties of theories. Particularly useful is an understanding of how each possible property of theories relates to empirical performance. A scientific community might identify some of the criteria most strongly diagnostic of high OS measures by considering what properties it seems minimally necessary or helpful for a theory to possess if it is to accord with large amounts of observational data. Among such properties might be consistency with known data, explanatory power, the ability to generate novel predictions, and as high as possible a degree of simplicity, as well as many properties of lesser scope peculiar to special sciences or topics. (One list of properties commonly held diagnostic of high OS measures is generated in NewtonSmith 1981, pp. 226-232.)

However, any mistakes made in describing the properties of the properties of theories will impair the effectiveness of scientists' evaluative criteria to diagnose high OS measures in theories. If a community believed that the property of, say, being consistent with atomism was necessary for a theory's truth, they would doubtless ensure that their criteria of theory-appraisal attached high value to it. If they were mistaken in their understanding of the properties of the property 'consistency with atomism', and this property did not in fact promote good empirical performance in theories, their criteria of theory-appraisal would be somewhat less strongly diagnostic of high OS measures than they might otherwise have been. 
Discovering the properties of the possible properties of theories is a task achieved by scientific communities only gradually, and by partly empirical methods, much like the task of discovering the properties of phenomena. Historians of science (e.g. Hesse 1980, pp. 14-15) often contrast late-twentieth-century beliefs about the properties of theories with the ideas prevalent in, say, the time of Copernicus, suggesting that there was then only rudimentary appreciation of what should be understood as a theory's empirical performance and what should count as a diagnostic indicator of this quality. Consistency with the Bible, with vitalism, with atomism, with common sense - these are possible properties of theories of which the properties have long been misunderstood and about which discoveries have gradually been made. Some discoveries about the properties of theories are counted among the most notable events in the history of science. For instance, certain sixteenth-century astronomers elucidated important scepticist implications of the property of 'consistency with bodies of known data', especially the fact that it can be found in theories which are false (Jardine 1987, pp. 90-92). The eighteenth-century mathematician Bayes, and others of his school, are credited with having discovered important properties of a theory's simplicity, especially its connection with the degrees of empirical support afforded to different theories by common bodies of data (Rosenkrantz 1976). In fact, much of the history of scientific method could be retold as a history of the gradual discovery of the properties of the possible properties of scientific theories.

There is no reason to suppose that this gradual process of discovery has now ceased. Indeed, some important discoveries about the properties of theories have occurred only very recently. For instance, the physics community has come to realize since the 1920 s, with the advent of quantum mechanics, that one possible property of theories, the property of being deterministic, lacks a property which had previously been ascribed to it: the property that any theory which is close to the truth must necessarily possess it.

Each successive discovery about the possible properties of theories has helped to improve the community's understanding of which properties are strongly diagnostic of high OS measures, and which are not. Before each discovery of this kind, the best available criteria of theoryappraisal were somewhat less strongly diagnostic of high OS measures. Because of this, the judgements made in the remote past about the OS measures of theories are in general not as reliable as those which take 
account of later discoveries about the properties of theories. It is only natural for those judgements to have been revised as further discoveries about the properties of theories accumulated.

Let us now turn back to the evidence constituted by radical transitions. According to the antirealist, radical transitions militate against realism because they show that, among the theories in science which have demonstrated a high degree of observational success, there are some which realists could not sensibly maintain were anything like close to the truth. This argument is now vulnerable to the following response, hinging on a reinterpretation of the historical data.

The only claims for which radical transitions can provide evidence are claims about some theories deemed successful in the history of science. Now, radical transitions took place within the sequence of discoveries of properties of the properties of theories, and before some such discoveries were made. Therefore, the theories deemed successful in the history of science were deemed to be so on the basis only of a set of criteria constructed in the light of imperfect knowledge about the properties of the properties of theories. These criteria are not direct checks of OS measures: they are only diagnostic of high OS measures. Moreover, some of them are only weakly diagnostic of high OS measures, so much so that they have since been repudiated by the scientific community as appropriate grounds for theory-evaluation.

But this means that Laudan's argument falls short of demonstrating that the earlier theories of radical transitions have high OS measures. It has therefore failed to show that scientific realism is untenable. The most ambitious claim that appeal to a particular radical transition can establish is that, among the theories to have received high scores on a set of criteria of theory-evaluation of the sort discussed above, there are some which realists would not be able to maintain were close to the truth. It is consistent with this claim to assert, as realism does, that a theory possessing the highest possible OS measure is true, or that theories possessing high OS measures are close to the truth, or even that a policy of choosing theories in virtue of their OS measures would result in the adoption of theories increasingly close to the truth.

The previous paragraph contains a minimal rebuttal of Laudan's argument: it points out that his case remains unproven, since we cannot know that the earlier theories of radical transitions have high OS measures, as his argument would require. But the resources of this approach enable us to go further, and achieve a more positive rebuttal. This 
further response consists of showing that, on the best available evidence, we must expect the OS measures of the earlier theories of radical transitions to be low, not high.

Since the earlier theory of the most recent radical transition was adopted, further discoveries have doubtless been made about the properties of theories. These latest discoveries have enabled the scientific community further to refine their criteria of theory-choice. As a consequence, we now possess a set of evaluative criteria which we have good reason to believe is more strongly diagnostic of high OS measures than were the sets in use up to the time of the most recent radical transition. This latest set of criteria attributes to all the theories which were "earlier theories' in radical transitions OS measures which are low, and much lower than those of the theories which succeeded them. (If this were not so, some of these discredited theories would constitute at least reasonably credible candidates for adoption today, while in fact they are not even remotely able to contend for adoption by the community - recall that we are speaking of such theories as the phlogiston and caloric theories.) This is our best available estimate of the OS measures of the theories abandoned in the course of radical transitions. Therefore, contrary to what Laudan suggests, radical transitions do not offer examples of theories which, while they cannot be considered close to the truth, have high OS measures: these theories have instead, to the best of our knowledge, low OS measures.

I believe that the realists' case can be reinforced further, by elucidating the nature of the historical variability of canons of theory-appraisal (section 4) and identifying radical transitions with revisions in these canons (section 5). First, however, we should consider what the antirealist might be tempted to respond.

3. SOME ANTIREALIST REJOINDERS AND THEIR SHORTCOMING

There are two immediate rejoinders to the argument of the previous section, by which the antirealist might attempt to show that radical transitions after all weigh against the plausibility of realism. I believe that each of them fails.

1. Antirealists might maintain the following: scientific realists have acquired a commitment to the claim that, if a theory scored high on some actual past criteria of theory-appraisal (not only "if it had a high OS measure"), it is close to the truth. Then radical transitions indeed 
militate against realism, since it is generally acknowledged by realists that the earlier theory in each transition, while highly-valued on evaluative criteria in force at its time, is not close to the truth.

The best realist response to this claim is flatly to deny its premise: realists have not (and certainly ought not to have) committed themselves to the claim that a theory which scored high on any old evaluative canon is close to the truth. In fact, this claim is of such extreme relativism that it is difficult to imagine its being seriously entertained by a realist: it amounts to claiming that a theory is close to the truth if at some point in history it was highly valued for being entrenched in Aristotelian scholasticism, or for according with a literalist reading of the bible, or for being supported by animism. All that the realist typically claims (or ought to claim) is some version of the tenet that theories with high OS measures are close to the truth.

2. The antirealist might maintain that the realist has acquired a commitment to the claim that those theories are close to the truth which have demonstrated to high degree a pragmatic 'manipulative efficacy' (an attainment different from observational success) in the surroundings of their adherents: Laudan $(1984 \mathrm{~b}$, p. 89) develops a notion of this kind. Radical transitions might then prove hostile to realism, if they were found to offer examples of theories which, while manipulatively very efficacious, could not reasonably be considered close to the truth.

The evident realist response is, again, that realists generally confine their claims to versions of the view that only those theories are close to the truth which have a high degree of observational success. To this may usefully be added the claim that, in so far as manipulative efficacy differs from observational success, there is an only loose correlation between a theory's proximity to the truth and its efficacy; in view of this, it is not surprising if some manipulatively efficacious theories are far from the truth.

Another possible response to this second antirealist rejoinder is the following. Laudan acknowledges (1984b, pp. 88-89) that 'manipulative efficacy' is a measure of success set out by the present-day philosopher, not a criterion of appraisal consistently employed throughout the history of science. This being so, the historical record cannot be presumed without further investigation to consist of a sequence of theories which were manipulatively very efficacious in the environment of their adherents. Such further investigation might be carried out in two ways: by 
measuring the efficacy of a theory either in the environment in which it was formulated and adopted, or in the environment of the present day. It is, for historiographic reasons (explored in e.g. Hesse 1980, pp. 23-25), difficult for us to establish the degree to which a theory was manipulatively efficacious in a past environment. That leaves the possibility of testing whether the earlier theory of some radical transition, such as the phlogiston theory, is manipulatively highly efficacious in the present-day environment. It would, I think, be plausible for realists to forecast that such theories would be judged manipulatively not very efficacious in our surroundings, especially if this test took the form (as do most practical theory-evaluations) of a comparison against an alternative theory, being the competing theory held to at the present day. If this judgement is borne out, the second possible antirealist rejoinder fares no better than the first.

\section{TRUTH IN THE LIMIT OF THEORY-CHOICE}

The argument has now reached the following state. It is admittedly implausible to assert of certain highly-valued theories in the history of science that they are close to the truth. From this, antirealists sought to conclude that realism has failed to establish an association between observational success and truth. Realists can now reply, however, that what ought throughout to have been claimed on behalf of the theories cited was not that they possessed high OS measures, but merely that they were deserving of adoption on the particular set of criteria of theory-appraisal being employed at the time of their formulation.

The question then arises: if, as I have argued, the policy of choosing theories in virtue of their OS measures is never actually applied in the history of science, what relation has the doctrine of realism to actual science? Scientific realism could be thought to reduce to the counterfactual claim that, if theory-choice were conducted on OS measures, the theories thereby adopted would approximate closely to the truth. But a more historicist answer will be preferred.

I claimed in section 2 that science's canons of theory-appraisal have shown considerable historical variability. Some of these changes were made by communities in the light of discoveries about the properties of the properties of theories. These discoveries led the community to compile a list of evaluative criteria which there is reason to believe was more strongly diagnostic of high OS measures in theories than was their 
previous list. (For discussions of the procedures by which empirical evaluative criteria can be appraised without circularity in the light of theories' empirical performance, see Rescher 1977, pp. 66-80, NewtonSmith 1981, pp. 224-225, and Laudan 1984a, pp. 42-66.)

Successive changes of this kind in a community's set of evaluative criteria can be interpreted as improvements of the fit between the sequence of theory-choices actually made by the community and the pattern of theory-choices as they might hypothetically be made on OS measures. In turn, the policy of choosing theories on OS measures can be described as the ideal limit of the series constituted by the policies of choosing theories on the basis of the community's successive actual sets of criteria of theory-evaluation.

This latter formulation offers a way to anchor the doctrine of realism to the progress of the actual history of science, while maintaining its links with the concept of high OS measures. I propose that scientific realism should be construed as making, in effect, the following claim: the policy of choosing theories for adoption, on the basis of a set of criteria which lies at the limit of the process of revision of the scientific community's set of criteria of theory-assessment, results in the adoption of theories close to the truth.

There are two comments on this formulation. First, by locating the object of their claims in the future, it has the advantage of absolving realists from having to profess belief in the proximity to the truth of every theory highly valued in past or current science. Secondly, it offers a new variant of the idea of 'convergent realism': while this doctrine has so far been interpreted most commonly as the theory-level claim that scientific theories are converging upon the truth, the present formulation advances the meta-level or methodology-level claim that actual criteria of theory-appraisal are converging on a limit constituted by the criterion of OS measures. (The notion of a limit of convergence at the level of theories is further explored, from a pragmatist perspective, by Jardine 1986.)

Those who believe that realism is akin to an empirical hypothesis (like Putnam 1978, pp. 78-79; for criticism see Laudan 1981, pp. 45-46) will ask whether this revised formulation of the doctrine is susceptible to test by appeal to the history of science. Perhaps it is. According to the realist, theories which possessed the highest OS measures possible would be true, since their claims would be true of all observable phenomena. If several theories each had the highest OS measures possible, 
and therefore were all true, they would be all consistent with one another. Therefore, if there were assured a supply of theories which were good enough, theory-choice on the basis of OS measures would lead to the adoption of theories which would be consistent with one another. It might be presumed that theory-choice on the basis of a set of criteria which approximates more and more closely to the criterion of OS measures would lead to the adoption of theories which exhibited a greater and greater degree of consistency with one another. Call the property of a number of theories of exhibiting a certain degree of consistency with one another their 'logical bunching'. Then a period of history of science, in which successive revisions were applied to the set of criteria of theory-choice to improve their approximation to OS measures, ought, if the doctrine of realism in this revised formulation is tenable, to exhibit an increased logical bunching of theories. The plausibility of scientific realism could therefore be investigated by comparing the degrees of logical bunching of theories in a science chosen under successive sets of evaluative criteria.

\section{A REALIST ACCOUNT OF RADICAL TRANSITIONS}

If formulated as above, the doctrine of scientific realism does not just accommodate the occurrence of radical transitions: it offers an account of them too. The account depicts radical transitions as coinciding with revisions of communities' criteria of theory-appraisal, of the sort described in the previous section.

Each radical transition, I claim, can be interpreted as an occasion in which the set of criteria of theory-assessment deemed by the community to be diagnostic of observational success underwent a change possessing two characteristics. The two characteristics are: the change was sufficient to compel or strongly encourage the abandonment of the theory which was, as a matter of fact, abandoned in the historical instance; and it left the community with a set of criteria which compelled or strongly encouraged the adoption of the theory which was, as a matter of fact, adopted as a result of the transition. (The reason for stipulating these characteristics is to prevent radical transitions from being linked with only cosmetic changes of evaluative criteria.) On this interpretation, a theory abandoned in a radical transition is abandoned on the grounds of its having fallen foul of some evaluative criterion newly (or simultaneously) introduced into the community's evaluative set; 
correspondingly, the theory embraced in a radical transition is one to which the new set of criteria led, or of which it permitted the adoption. (This suggestion is reminiscent of the idea of Kuhn 1962, pp. 109110 , that a paradigm comes into the world equipped with criteria of assessment on which its theories will achieve scores that are high, and higher than those of their predecessors.)

These changes of evaluative criteria should be imagined not as occurring gratuitously, but rather as being prompted by new discoveries about the possible properties of theories, as described in section 2 . The revisions of the community's evaluative canon are signs of the community's wish to take the fullest account in its theory-choices of its improved understanding of the properties of the properties of theories.

There is an easy defence for the claim that every radical transition can be interpreted as a case in which the community's set of criteria for theory-choice underwent a change possessing the two characteristics cited. A radical transition is defined as an episode in which one theory is succeeded by another containing radically different theoretical terms. If the terms of the later theory are so different from those of its predecessor, the theoretical terms of the later theory will possess some characteristics not possessed by the theoretical terms of the earlier theory, and vice versa. The abandonment of the earlier theory and the adoption of the later one can be compelled or strongly encouraged by criteria of theory-choice which refer appositely to these disjoint sets of features. In general, therefore, the possibility of associating any radical transition with a change of evaluative canon of this kind is not in doubt.

For instance, the transition from the phlogiston to the oxygen theory can be interpreted as a case in which the scientific community changed its mind about the sorts of theories which could legitimately be proposed to solve empirical problems, or in which it revised the portfolio of criteria which it deemed to be diagnostic of high OS measures. The community may be interpreted as having come to hold to, say, the criterion that a chemical theory ought not to invoke substances (or, as the antirealist would put it, theoretical terms which might be construed as a description of substances) which possessed the properties of impalpability or negative weight. The phlogiston theory did not, and the oxygen theory did, satisfy this criterion for theory-choice: this is why - the realist reading continues - the oxygen theory was preferred to the phlogiston theory from a certain date onwards.

While the previous paragraph was meant only to convey the sort of 
construction which the realist would seek to place upon a radical transition, recent historiography of the chemical revolution (see Donovan 1988 , pp. 228-231) has in fact drawn attention to the influence which changes in criteria of theory-appraisal exerted upon changes in substantive theory. A suggestion which has gathered particular support is that Lavoisier should be seen as having imported into chemistry standards of appraisal characteristic of mathematical physics, and that these were in part responsible for dissatisfaction with phlogiston theory (Melhado 1985, pp. 209-211; Donovan 1988, pp. 220-222). Rather than its being the case that "after discovering oxygen Lavoisier worked in a different world", as Kuhn (1962, p. 118) anti-realistically puts it, Lavoisier and his community embraced the oxygen theory because their world had already changed in one particular respect: in the respect of coming to contain a set of criteria for theory-choice in chemistry different from the one upon which the phlogiston theory had previously been valued.

Similarly, the disappearance of the caloric from theories of heat in the 1810 s can be retraced in part to changes in methodological canons, as a result of which scepticism arose about the imponderable fluids traditionally postulated in Laplacian physics. (On this interpretation of the fall of the caloric theory, see Fox 1990, pp. 287-289.)

If this interpretation of radical transitions is justified, these instances ought to be thought of less as prompted by discoveries about the properties of phenomena, and more as prompted by discoveries about the possible properties of theories. Radical transitions, as opposed to more mundane cases of theory-succession, might signal to the historian of science times at which a new discovery or understanding about the properties of the properties of theories was first applied in theoryappraisal.

\section{THE REALIST'S ANSWER}

Realists have acquired a new response to antirealists. The latter had asked them, "How do you explain the failure to refer of the theoretical terms of theories such as the phlogiston theory, if a) you believe that observational success is linked to truth in the way you require, and b) you admit that the phlogiston theory was successful?" The antirealists' arguments have aimed to force realists to the admission, "The notion that theoretical terms should be interpreted as referring is incoherent." The realist is by contrast now in a position to answer as follows: "The 
phlogiston theory demonstrated to the eighteenth-century community its empirical value upon being judged on such-and-such a set of criteria. This set of criteria was then deemed diagnostic of high OS measures, but is different both from our present set of criteria of theory-appraisal, and (even more emphatically) from a criterion of directly consulting theories' OS measures. Therefore, the phlogiston theory never demonstrated to the eighteenth-century community that it possessed a high OS measure. Part of the reason why we have since come to realize that the central term of the phlogiston theory fails to refer, and therefore that the theory is far from the truth, is that we now evaluate theories on this such-and-such other set of criteria of theory-appraisal, the verdicts of which we deem to be a closer approximation to OS measures. This is also part of the reason why the present-day chemical community no longer holds to the phlogiston theory."

On this interpretation, far from confuting scientific realism, casestudies of radical transitions can offer evidential support to the doctrine: they are capable of confirming that in radical transitions criteria of theory-choice undergo profound revision, and that therefore the theories embraced at the eve of a transition were chosen for adoption on criteria different from those applied upon its completion, and certainly different from OS measures. Such findings would substantiate one of the theses defended here: that the reason why certain highly-valued past theories can be so obviously far from the truth is not that scientific realism is incoherent, but that theories are in fact valued on grounds other than their possession of high OS measures.

I offer this paper as a response to a particular criticism of scientific realism, rather than as an overall vindication of the doctrine. Clearly, there may still be grounds on which to doubt the plausibility of realism; but I believe that arguments based on the evidence from radical transitions, which I reconstructed in section 1, are not among them.

\section{ACKNOWLEDGEMENTS}

An abridged version of this paper was given at the conference on Recent Advances in the Philosophy of Science held at the University of Amsterdam in August 1991. I am grateful to the participants for their comments. 


\section{REFERENCES}

Boyd, R. N.: 1984, 'The Current Status of Scientific Realism', in Leplin (ed.) 1984, pp. 41-82.

Brown, J. R.: 1985, 'Explaining the Success of Science', Ratio 27, 49-66.

Buchdahl, G.: 1970, 'History of Science and Criteria of Choice', in R. H. Stuewer (ed.), Historical and Philosophical Perspectives of Science, Minnesota Studies in the Philosophy of Science, Vol. 5, University of Minnesota Press, Minneapolis, pp. 204230.

Churchland, P. M.: 1985, 'The Ontological Status of Observables: In Praise of the Superempirical Virtues', in P. M. Churchland and C. A. Hooker (eds), Images of Science: Essays on Realism and Empiricism, University of Chicago Press, Chicago, pp. $35-47$.

Cummiskey, D.: 1992, 'Reference Failure and Scientific Realism: A Response to the Meta-induction', British Journal for the Philosophy of Science 43, 21-40.

Donovan, A.: 1988, 'Lavoisier and the Origins of Modern Chemistry', Osiris, second series, 4, 214-231.

Fox, R.: 1990, 'Laplacian Physics', in Olby et al. (eds) 1990, pp. 278-294.

Hardin, C. L. and Rosenberg, A.: 1982, 'In Defense of Convergent Realism', Philosophy of Science 49, 604-615.

Hesse, M. B.: 1980, Revolutions and Reconstructions in the Philosophy of Science, Harvester Press, Brighton.

Jardine, N.: 1986, The Fortunes of Inquiry, Clarendon Press, Oxford.

Jardine, N.: 1987, 'Scepticism in Renaissance Astronomy: A Preliminary Study', in R. H. Popkin and C. B. Schmitt (eds.), Scepticism from the Renaissance to the Enlightenment, Harrassowitz, Wiesbaden, pp. 83-102.

Kuhn, T. S.: 1962, The Structure of Scientific Revolutions, University of Chicago Press, Chicago (Second edition, 1970.)

Laudan, L.: 1981, 'A Confutation of Convergent Realism', Philosophy of Science 48, 19-49; reprinted in Leplin (ed.) 1984, pp. 218-249.

Laudan, L.: 1984a, Science and Values: The Aims of Science and Their Role in Scientific Debate, University of California Press, Berkeley.

Laudan, L.: 1984b, 'Explaining the Success of Science: Beyond Epistemic Realism and Relativism', in J. T. Cushing, C. F. Delaney and G. M. Gutting (eds.), Science and Reality: Recent Work in the Philosophy of Science, University of Notre Dame Press, Notre Dame, Ind., pp. 83-105.

Leplin, J. (ed.): 1984, Scientific Realism, University of California Press, Berkeley.

McMullin, E.: 1984, 'A Case for Scientific Realism', in Leplin (ed.) 1984, pp. 8-40.

Melhado, E. M.: 1985, 'Chemistry, Physics, and the Chemical Revolution', Isis 76, $195-$ 211.

Miller, R. W.: 1987, Fact and Method: Explanation, Confirmation and Reality in the Natural and the Social Sciences, Princeton University Press, Princeton, N.J.

Newton-Smith, W. H.: 1981, The Rationality of Science, Routledge and Kegan Paul, London.

Newton-Smith, W. H.: 1990, 'Realism', in Olby et al. (eds.) 1990, pp. 181-195.

Olby, R. C., Cantor, G. N., Christie, J. R. R., and Hodge, M. J. S. (eds): 1990, Companion to the History of Modern Science, Routledge, London. 
Putnam, H.: 1978, Meaning and the Moral Sciences, Routledge and Kegan Paul, London. Rescher, N.: 1977, Methodological Pragmatism: A Systems-Theoretic Approach to the Theory of Knowledge, Blackwell, Oxford.

Rosenkrantz, R. D.: 1976, 'Simplicity', in W. L. Harper and C. A. Hooker (eds.), Foundations of Probability Theory, Statistical Inference, and Statistical Theories of Science, Three volumes, Reidel, Dordrecht, vol. I, pp. 167-196.

Thagard, P.: 1988, Computational Philosophy of Science, MIT Press, Cambridge, Mass. van Fraassen, B. C.: 1980, The Scientific Image, Clarendon Press, Oxford.

Faculty of Philosophy

University of Leiden

P.O. Box 9515

2300 RA Leiden

The Netherlands

Manuscript submitted September 25, 1991

Final version received June 29, 1992 